\title{
A Framework for the Generation of Realistic Synthetic Cardiac Ultrasound and Magnetic Resonance Imaging Sequences from the same Virtual Patients
}

\author{
Y. Zhou, S. Giffard-Roisin, M. De Craene, S. Camarasu-Pop, J. D’hooge, M. Alessandrini, D. Friboulet, \\ M. Sermesant, and O. Bernard
}

\begin{abstract}
The use of synthetic sequences is one of the most promising tools for advanced in silico evaluation of the quantification of cardiac deformation and strain through 3D ultrasound (US) and magnetic resonance (MR) imaging. In this paper, we propose the first simulation framework which allows the generation of realistic 3D synthetic cardiac US and MR (both cine and tagging) image sequences from the same virtual patient. A state-of-the-art electromechanical (E/M) model was exploited for simulating groundtruth cardiac motion fields ranging from healthy to various pathological cases including both ventricular dyssynchrony and myocardial ischemia. The E/M groundtruth along with template MR/US images and physical simulators were combined in a unified framework for generating synthetic data. We efficiently merged several warping strategies to keep full control of myocardial deformations while preserving realistic image texture. In total, we generated 18 virtual patients, each with synthetic 3D US, cine MR and tagged MR sequences. The simulated images were evaluated both qualitatively by showing realistic textures and quantitatively by observing myocardial intensity distributions similar to real data. In particular, the US simulation showed a smoother myocardium/background interface than the state-of-the-art. We also assessed the mechanical properties. The pathological subjects were discriminated from the healthy ones by both global indexes (ejection fraction and the global circumferential strain) and regional strain curves. The synthetic database is comprehensive in terms of both pathology and modality, and has a level of realism sufficient for validation purposes. All the 90 sequences are made publicly available to the research community via an open-access database.
\end{abstract}

Index Terms-Multimodal cardiac imaging, numerical simulation, cardiac strain, motion estimation, synthetic sequences, electromechanical model

\section{INTRODUCTION}

Y. Zhou, S. Camarasu-Pop, D. Friboulet and O. Bernard are with the University of Lyon, CREATIS, CNRS UMR5220, Inserm U1044, INSA-Lyon, University of Lyon 1, Villeurbanne, France. E-mail: olivier.bernard@creatis.insalyon.fr

Y. Zhou and M. De Craene are with Philips Research Paris (Medisys), Suresnes, France

S. Giffard-Roisin and M. Sermesant are with the Inria-Asclepios Project, Sophia Antipolis, France

J. D'hooge is with the Lab on Cardiovascular Imaging \& Dynamics, Department of Cardiovascular Sciences, KU Leuven, Belgium

M. Alessandrini is with the Department Cardiovascular Sciences, Laboratory of Cardiovascular Imaging and Dynamics, KU Leuven, Leuven, Belgium $\&$ the Department of Electrical, Electronic and Information Engineering, University of Bologna, Bologna, Italy
$\mathbf{C}$ ARDIOVASCULAR diseases remain the number one cause of death globally [1]. Detecting the diseases at an early stage helps reducing the mortality rate. In recent years, the diagnosis of cardiovascular diseases has been much improved by the development of cardiac imaging techniques. Analyzing cardiac motion and strain from medical images reveals important clinical implications such as ischemia or dyssynchrony. Important advances have been achieved in both ultrasound (US) and magnetic resonance (MR) technologies in recent decades. As for US, rapid 3D volumetric imaging is now feasible, thanks to the introduction of 2D matrix array transducer technology [2]. Also, ultrasound is portable and non-invasive, being suitable for providing bed-side care to patients. Cardiac cine MR is widely used in clinical routine for evaluating global cardiac function since it provides clear contrast between the myocardium and the surrounding tissues. Aside from cine, tagging is the MR sequence that provides the most detailed information about local cardiac deformations [3]. It is considered as the gold standard for quantifying local myocardial motion and strain. Nonetheless, the use of tagged MR in clinical practice is somewhat held back by the lack of reliable automatic post-processing tools [4], as well as its limited spatial resolution for distinguishing structures [5].

Despite all those developments of imaging techniques, the assessment of cardiac function generally relies on global measures, such as left ventricle (LV) ejection fraction (EF) and global longitudinal strain (GLS) [6]. GLS quantified from speckle tracking echocardiography [7] is currently the best evaluated strain parameter [8] [9]. Compared with global strains, regional strains are less reliable and reproducible as reported in [9] [10] [11]. Consequently, regional strain analysis is currently applied as a supplementary diagnosis method, but is still not ready for full clinical implementation in spite of their potential impact in many applications [6] [12]. One of the reasons is the fact that a well-established validation of regional strain imaging is still missing at the moment, despite the existence of a large number of dedicated quantification algorithms both in US [13] and tagged MR [14]. A comprehensive and thorough quantitative validation

Copyright (c) 2017 IEEE. Personal use of this material is permitted. However, permission to use this material for any other purposes must be obtained from the IEEE by sending a request to pubs-permissions@ieee.org. 
of these methods based on dense strain measurements would thus represent a major progress.

Several groups already made some initial attempts towards this objective. Synthetic US [2] [18] [19], cine MR [18] [19] and tagged MR [20] [21] images were generated. They all adopted computer-generated synthetic sequences for validating motion tracking for one specific image modality. The interest in using synthetic images has been well detailed in several studies [2] [18]. Their main advantage is the straightforward possibility of assessing the accuracy of algorithms by comparing their performance against the controlled (and thus known) ground-truth deformations. In this work, instead of simulating a single modality, we opted for generating multimodal images for the same virtual subject. This allows for a direct comparison of different imaging modalities. For instance, there is currently increasing research interest in investigating whether cine MR feature tracking (CMR-FT) [15] can quantify strain values similar to classical techniques applied on tagged MR [16] and US data [17]. Although the corresponding studies showed a close correlation between CMR-FT results and the ones obtained from tagged MR and US, it is still not clear which modality outperforms the others, in which aspects and to which extent. Thus, providing the research community with a multimodal synthetic cardiac database is of great value in pushing forward not only the validation within a single modality but also the cross-modality comparison. Nonetheless, the utility of such synthetic images depends heavily on the realism of the simulations. Lots of efforts have been made this last decade to improve the realism of cardiac synthetic images in each modality, as described hereunder.

\section{A. Cine MR image simulation}

Tobon-Gomez et al. [22] and Wissmann et al. [23] simulated cardiac cine MR image sequences based on the XCAT [24] phantom. In [22], a tissue segmentation (or labeling) was performed where different tissue types were associated with relevant $\mathrm{T} 1, \mathrm{~T} 2$ and proton density (PD) values obtained from the literature. The static T1, T2 and PD maps at end-diastole (ED) were then warped by cardiac motion obtained from the XCAT phantom. Finally, at each time frame, an MR simulator was used to generate the cine MR images. Wissmann et al. [23] directly simulated image intensities by signal equations instead of using an MR simulator. They claim that this choice facilitates the simulation of sampling strategies, trajectory optimization and post-processing methods [23]. Haddad et al. [25] and Glatard et al. [19] also synthesized 3D+t cardiac cine MR images. They adopted a similar approach to that presented in [22] by combining a beating heart model with an MR simulator. The main difference is in the heart model, which is extracted from real acquisitions as described in [26]. Recently, Prakosa et al. [18] developed a pipeline that combines an electromechanical model (E/M) of the heart with template acquisitions for generating realistic cine MR data. The E/M model has the benefit of relating the contraction law with its biophysical causes which allows for a more realistic incorporation of physiological and pathological conditions. The template cine MR sequence was registered to the first frame, yielding a "frozen" sequence. This sequence was then warped with the simulation results to create synthetic images.

\section{B. Tagged MR image simulation}

Compared to cine MR, little work has been done in simulating cardiac tagged MR. Crum et al. [27] simulated the LV in short axis slices. They modeled the corresponding anatomy using a simple ring shape. Using a motion directly computed from a real cine MR sequence, the authors warped the initial simulated ED image to the rest of the sequence. The tagging pattern was simulated by applying a sinusoidal modulation function in the spatial domain. Later in [28], Crum et al. improved the generation of tag intensity profiles by using a frequency-domain model. Similarly, Waks et al. [29] used a prolate sphere to mimic the LV geometry and a 13parameter kinematic motion model. The model parameters were determined by a least-squares fit to the displacements of the implanted markers tracked from a dog heart acquisition. Sermesant et al. [30] segmented myocardium from a real tagged MR image and further added tag lines to the binary mask. Finally, this image was warped by cardiac motions generated by the E/M model. Clarysse et al. [20] warped a real short-axis tagged MR image by a simple kinematic mode-based heart motion model. However, the motion model was too simplistic to represent the complexity of true heart motion. Similarly, in our previous work presented in [21], a normal cardiac motion pattern was first simulated by the $\mathrm{E} / \mathrm{M}$ model, and a real acquisition was further deformed for assigning realistic image intensities. However, only one tagged MR sequence of a healthy heart was simulated.

\section{Echocardiography image simulation}

As for the simulation of echocardiography images, Alessandrini et al. recently gave a rather comprehensive analysis in [2]. We provide here a brief summary of the main techniques. Several groups already used the E/M model for generating synthetic cardiac deformation fields in US [31] [18] [2]. Prakosa et al. [18] proposed a warping-based method. The template sequence was registered to the E/M simulations. Although the resulting images showed realistic textures, the warping artifacts undermined somehow the level of realism. To alleviate warping artifacts, De Craene et al. [31] used a physical ultrasound simulator for the image generation. However, the underlying speckle model was too simplistic (i.e. high speckle intensity inside the muscle and low intensity outside), yielding unrealistic binary-like synthetic US images. Recently, Alessandrini et al. [2] combined the E/M model, physical ultrasound simulator and template US recordings into a unified pipeline. Their simulation pipeline combined the advantages of both [18] and [31] and was able to produce realistic synthetic images. However, the transition between the myocardium and the surrounding tissues was not sufficiently controlled, as discussed in [2], reducing the degree of realism of the simulated sequences. 


\section{Contributions of the paper}

In this study, we extended the pipeline initially proposed in [2] for US to MR modality in order to generate realistic 3D cardiac cine MR, tagged MR and echocardiography sequences from the same virtual patient. The proposed simulation pipeline consists of three main elements: i) use of real clinical recordings to extract relevant information to improve the realism of the generated images; ii) involvement of an E/M model of the heart [32] to generate healthy and pathological synthetic motion field used as reference; iii) use of physical simulation environments to model image formation with the possibility to introduce complex effects such as tag fading for tagged MR. The proposed extended pipeline was used to create a database of 18 virtual patients including healthy and various pathological cases, i.e. ischemia and dyssynchrony. For each patient, benchmark sequences of 3D US, cine and tagged MR were generated. We generated a total of 90 synthetic sequences representing 2700 image volumes. The synthetic sequences along with the corresponding reference motion fields are made publicly available via an open-access database ${ }^{1}$. The novelties introduced in this paper are the following ${ }^{2}$ :

- The combination of an E/M model with an MR physical simulator to introduce the possibility of interacting with the image formation process to introduce complex effects such as tag fading;

- The use of multimodal template sequences from the same patient to extract the most relevant information from each modality (e.g. anatomical structures from cine and motion estimation from tagged MR) in order to generate both cine and tagged synthetic sequences;

- The introduction of a novel combinative warping strategy, with the goal of reducing motion artifacts that may occur in myocardium regions;

- The contribution of the first unified framework which allows the generation of multimodal (US, cine and tagged MR) realistic synthetic sequences for the same virtual patient;

- The use of a novel strategy based on a Gaussian weighting function to generate a smooth interface between the myocardium and the surrounding structures.

The remainder of the paper is organized as follows. In Section II, the proposed generic pipeline is described. The quality of the simulated sequences for MR modalities is then investigated in Section III. A discussion part is provided in Section IV while concluding remarks are given in Section V.

\section{Methodology}

We extend in this section the ultrasound pipeline initially developed in [2] to the generation of synthetic MR sequences. The interest is to derive a generic framework which can be used to simulate multimodal (US, cine and tagged MR) cardiac

\footnotetext{
${ }^{1}$ http://humanheart-project.creatis.insa-lyon.fr/multimodalityStraus.html

${ }^{2} \mathrm{~A}$ preliminary version of this work appeared in [21]. While in [21] only a first attempt to generate realistic tagged MR is reported, the present paper describes in details a more advanced pipeline which integrates cine MR and US in a unifying framework. It is to the best of our knowledge the first time that multimodal cardiac synthetic sequences of the same virtual patient can be generated with realistic textures in each modality.
}

sequences for the same virtual patient. Since much of the innovations presented in this work reside on the MR aspects, we mainly focus in this section on the different steps we introduced to simulate realistic cine and tagged MR sequences. It is worth pointing out that the same pipeline was also used in this paper to generate synthetic US images, as shown in Section III (only the "MR Modeling" block has to be replaced by the one proposed in [2]).

A schematic view of the pipeline extended to MR is shown in Fig. 1. Each block specifies the number of the section where it is described in detail. Briefly, real acquisitions (cine/tagged MR) are used as template sequences (Sect. II-A) for deriving realistic textures. The first step is to segment the right ventricle (RV) and the left ventricle (LV) on the first frame of cine MR template sequence (Sect. II-B1). The E/M model is then applied to the segmented 3D geometry to simulate one cardiac cycle (Sect. II-C1). For each frame of the simulated sequence, cine or tagged MR images are generated from a physical simulator which takes relaxation times $T_{1}$ and $T_{2}$ and proton density maps as inputs (Sect. II-B). To achieve realistic texture, proton density maps are derived from the template recordings. This requires a dedicated spatio-temporal alignment between the simulations and the template recordings (Sect. II-D). As such, the pipeline will generate synthetic cine and tagged MR sequences with a fully controlled deformation field and with a texture visually similar to the one of the corresponding template recording.

\section{A. Template image sequences}

3D template recordings used in this study come from the open access database provided in [33]. For each volunteer, one US, one cine MR and three tagged MR sequences were acquired. The MR datasets were acquired using a 3T Philips Achieva System (Philips Healthcare, Best, The Netherlands). The MR sequences processed by the participants were cine steady state free precession (SSFP) [34] and 3D tagged MR (3DTAG) [35]. Cine MR images were acquired during breathholds of approximately 15 seconds and were gated to the vector ECG. 3DTAG datasets were obtained with three sequential breath-hold acquisitions in each orthogonal directions. Since MR images were acquired in the same patient coordinate system, cine and tagged MR sequences are naturally aligned in space. A set of 24 manual landmarks identifying the same keypoints in all the modalities allows the derivation of a simple rigid transformation to pass from MR to US coordinates. These landmarks are evenly distributed over the LV, with 8 landmarks at each of the three ventricular levels (basal, midventricular, apical) [33]. All the acquisitions represent one cardiac cycle. The US template has a spatial resolution of $0.82 \times 0.83 \times 0.73 \mathrm{~mm}^{3}$ while the cine and tagged MR have spatial resolutions of $1.25 \times 1.25 \times 8.08 \mathrm{~mm}^{3}$ and $0.96 \times 0.96 \times 7.71 \mathrm{~mm}^{3}$.

\section{B. MR modeling}

We used the ODIN simulator [36] to generate both cine and tagged MR images. ODIN is a physical simulator which allows either the selection or the design of specific MR 


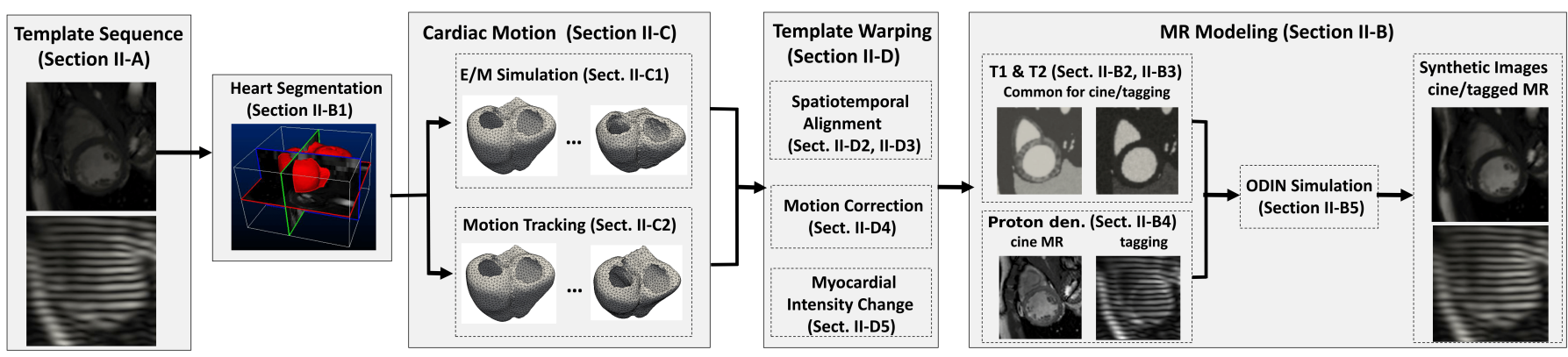

Fig. 1: Proposed pipeline for the simulation of realistic cardiac cine and tagged MR sequences.

sequences. To simulate one 3D image, ODIN requires $T_{1}, T_{2}$ and proton density maps as inputs. In this study, we used the same $T_{1}$ and $T_{2}$ maps to generate both cine and tagged MR sequences for each virtual patient, only the proton density maps were computed separately for each modality and for each time instant of the simulated sequence. The first step of the proposed MR pipeline is the computation of the initial $T_{1}$ and $T_{2}$ maps for the first frame. We first describe how these initial maps were generated. We then give details on the way they were extended to all time frames.

1) Heart segmentation: Because we assign different $T_{1} / T_{2}$ values per structure, we first need to segment the initial template image into different classes, i.e. myocardium, blood, lung and air. Since cine MR provides the best image contrast and since all the template modalities can easily be mapped, it represents a natural choice to extract the heart geometry. We first used the VP2HF segmentation pipeline proposed in [37] to segment the myocardium and the underlying blood pools. We then segmented the lung and the air outside the body thanks to simple mathematical morphology operations. Finally, the remaining unlabeled pixels were classified by thresholding: high intensity pixels belonging to blood and the rest to muscles. Fig. $2 \mathrm{~b}$ shows a typical segmentation result obtained by using such procedure.

2) Initial $T_{1}$ and $T_{2}$ maps: We assigned $T_{1}$ and $T_{2}$ maps using published reference mean and standard deviation values (denoted as $u$ and $\sigma$ ) available from [22]. At each pixel location, $T_{1}$ and $T_{2}$ values were randomly sampled from Gaussian distributions, yielding initial static maps denoted as $T_{1}^{0}$ and $T_{2}^{0}$ (see Fig. 2c and 2d). The relevant $u$ and $\sigma$ values used in this study are listed in Table I. Moreover, since the myocardium is our region of interest, a dedicated strategy was applied to better control its underlying texture. Indeed, since tagged MR intensity changes contain strong $T_{1}$ information, it is possible to deduce relevant local and structural $T_{1}$ values from image intensities. In particular, temporal intensity changes of the CSPAMM tagged MR sequence can be described as follows [38] [39]:

$$
I_{\text {tag }}^{n}=I_{\text {tag }}^{0} \exp \left\{-\frac{n \Delta t}{T_{1}}\right\} \frac{\sin \left(\alpha_{n}\right)}{\sin \left(\alpha_{0}\right)} \prod_{j=0}^{n-1} \cos \left(\alpha_{j}\right), \quad \forall n \geq 1
$$

where $\Delta t$ is the temporal resolution, $n$ is the frame index and $I_{\text {tag }}^{n}$ is the tagging intensities of the material point at frame
TABLE I: $T_{1}$ and $T_{2}$ reference values attached to each label of the segmented cine MR image.

\begin{tabular}{ccccccc}
\hline \multirow{2}{*}{ Label } & \multirow{2}{*}{ Class } & \multicolumn{2}{c}{ T1 (ms) } & & \multicolumn{2}{c}{ T2 (ms) } \\
\cline { 3 - 4 } \cline { 6 - 7 } & & $u$ & $\sigma$ & & $u$ & $\sigma$ \\
\hline 0 & Air & 0 & 0 & & 0 & 0 \\
1 & Blood & 1516 & 21 & & 189 & 26 \\
2 & Myocardium & 982 & 46 & & 54 & 12 \\
3 & Lung & 1199 & 117 & & 79 & 29 \\
4 & Other (low) & 549 & 52 & & 49 & 20 \\
5 & Other (high) & 1516 & 21 & & 189 & 26 \\
\hline
\end{tabular}

n. $\alpha_{n}$ corresponds to the RF flip angle at frame $n$ and can be easily computed from the method described in the appendix. Eq. (1) thus gives access to $T_{1}$ information from the intensity changes of the tagged MR template sequence. In order to accurately estimate the $T_{1}$ map of the myocardium, the following procedure was repeated for all pixels belonging to this region. For a given point at ED, we tracked it through the entire tagged MR template sequence (this step is later described in Section II-C2). This gives a sequence of intensities $I_{t a g}^{n}$ for all time instants. The corresponding $T_{1}$ value was then estimated from linear least square minimization problem:

$$
\arg \min _{T_{1}} \sum_{n}\left(\log \left(\frac{I_{t a g}^{n} \sin \left(\alpha_{0}\right)}{I_{\text {tag }}^{0} \sin \left(\alpha_{n}\right) \prod_{j=0}^{n-1} \cos \left(\alpha_{j}\right)}\right) T_{1}+n \Delta t\right)^{2}
$$

Since there are three template sequences of tagged MR images per subject (Section II-A), we derived three different $T_{1}$ values for the same myocardium point. A median filtering was then applied for removing possible outliers, yielding the final $T_{1}^{0}$ myocardial map as shown in Fig. 2c.

3) Dynamic $T_{1}$ and $T_{2}$ maps: Since each frame of the simulated sequences was generated independently, the initial $T_{1}^{0}$ and $T_{2}^{0}$ maps previously described had to be extended over time. This was achieved by a dedicated warping strategy described in Section II-D. The corresponding dynamic maps are denoted as $T_{1}^{k}$ and $T_{2}^{k}, k$ indexing the simulation frame.

4) Proton density maps: For each simulation frame, we further computed the proton density map from the corresponding template recordings. This ensures the generation of a texture 


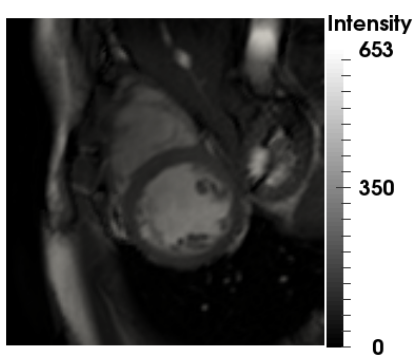

(a) cine $\mathrm{MR}$ at $\mathrm{ED}$

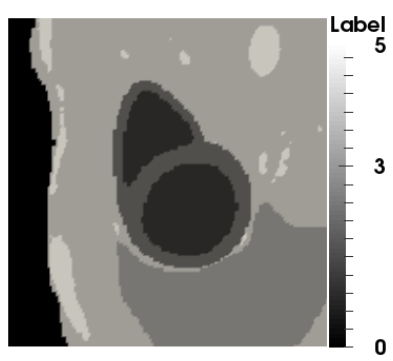

(b) labeling/segmentation

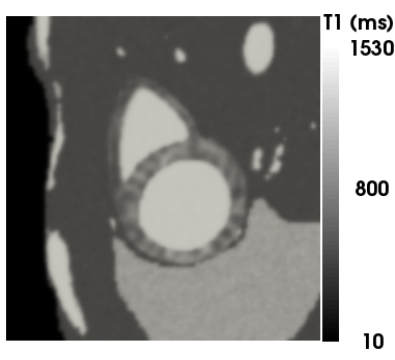

(c) $T_{1}^{0}$

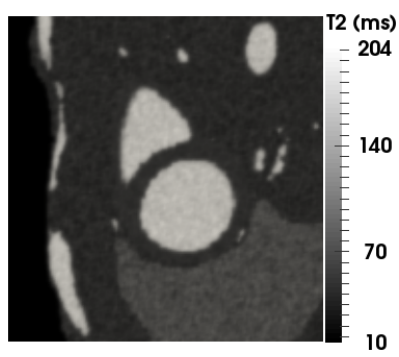

(d) $T_{2}^{0}$

Fig. 2: Illustration of the different steps involved to simulate the initial $T_{1}$ and $T_{2}$ maps relative to the first frame (ED).

visually similar to the one of the underlying template. To this aim, one needs to spatially and temporally align each frame of the simulated sequence with its counterpart in the template sequence. This was achieved by a dedicated warping strategy detailed in Section II-D. The outputs of this step correspond to a set of warped templates denoted as $\tilde{I}_{\text {cine }}^{k}$ and $\tilde{I}_{\text {tag }}^{k}$ and aligned with each simulation frame $k$.

Cardiac cine MR images were acquired using the balanced steady state free precession (bSSFP) MR sequence [34]. The proton density map $\rho_{\text {cine }}^{k}$ of frame $k$ can then be computed from the corresponding warped template [34]:

$$
\rho_{\text {cine }}^{k}=\tilde{I}_{\text {cine }}^{k} \sqrt{T_{1}^{k} / T_{2}^{k}}
$$

For tagged MR, the characteristics of the CSPAMM sequence provide the following $\rho_{t a g}^{k}$ map [39]:

$$
\rho_{t a g}^{k}=\tilde{I}_{t a g}^{k} \exp ^{\frac{T E}{T_{2}^{*}}} / \sin \left(\alpha_{k}^{s}\right)
$$

where $\alpha_{k}^{s}$ corresponds to the simulated RF flip angle, where $T E$ is the echo time here set to $21 \mathrm{~ms}$. $T_{2}^{*}$ corresponds to the effective spin-spin relaxation times and are here approximated by the relaxation time $T_{2}$ [40]. We thus assume $T_{2}^{*}=T_{2}^{k}$ in the sequel.

5) ODIN simulation: Each frame $k$ of the simulated sequences was independently generated from ODIN by using the $T_{1}^{k}, T_{2}^{k}$ and $\rho_{\text {mod }}^{k}$ maps derived above (with $\bmod =$ cine or $t a g$ ) as inputs. For the sake of simplicity, we decided to use the same resolution for those maps and the generated output MR images. Moreover, since we wanted to reproduce as close as possible the template recordings, we used the image properties (origin, spacing, size and axis orientations) of the template cine and tagged MR as reference. This defines the pixel positions of the simulated images for each modality. The $T_{1}^{k}, T_{2}^{k}$ and $\rho_{\text {mod }}^{k}$ maps were thus interpolated to those pixel positions before being passed on to ODIN. We used respectively the odinfisp and odinepi MR sequences available under ODIN for simulating cine and tagged MR images. Indeed these sequences are consistent with the corresponding real acquisition protocol, i.e. the bSSFP for cine sequence [33] and the EPI for tagged sequence [35].

\section{Cardiac motion}

As commented in Section II-B1, the first frame of the template cine MR sequence was segmented into different classes including myocardium region ( $\mathrm{LV}$ and $\mathrm{RV}$ ). From this mask, a volumetric mesh (both LV and RV) was generated using [2] and propagated to all further simulation and template image time frames as described hereinafter.

1) $E / M$ simulation: From the myocardial mask of the first frame, a tetrahedral mesh was generated using the $\mathrm{CGAL}^{3}$ meshing software [41] and passed to the E/M simulator which generated as output a sequence of volumetric meshes $\mathcal{S}_{k}$. The E/M simulator applies the Bestel-Clement-Sorine model which estimates the electrical activation and the resulting mechanical contraction [32] and is implemented in the SOFA framework ${ }^{4}$. This model was chosen for its realistic properties and it complies with the four cardiac phases (isovolumic contraction, ejection, passive and active filling). Cardiac fiber orientations were estimated with a rule-based method (elevation angle between $-70^{\circ}$ to $+70^{\circ}$ ) in order to model the E/M anisotropy. It has already shown its efficiency in the generation of realistic US sequences [2]. A number of biophysical parameters such as the local myocardial contractility, stiffness and conduction governs the $\mathrm{E} / \mathrm{M}$ equations. By varying those parameters, both normal and pathological cardiac motion fields can be simulated. For each patient, we generated in this study one healthy, one dyssynchrony (LBBB) and four realistic ischemic hearts. For the dyssynchony, only the right endocardium was initially activated. For the ischemia, varying scar locations with random and realistic shapes were simulated [42] in parts of the LV where arterial occlusion can occur: one for the Left Anterior Descending artery (LAD), one for the Left Circumflex (LCX), and two for Right Coronary Artery (RCA). Corresponding figures could be found in the supplementary materials (available in the supplementary files /multimedia tab).

2) Motion Tracking: Since the cine and tagged MR were acquired under the same coordinate system, we used the tagged MR sequence to perform the tracking. The LV and $\mathrm{RV}$ of the first E/M model mesh $\mathcal{S}_{0}$ (the E/M model and the template are naturally aligned at the first frame) were extracted and processed separately by two state-of-the-art algorithms in order to maximize the tracking accuracy. The LV border was tracked using the HarpAR algorithm given its good ability

\footnotetext{
${ }^{3}$ the open source Computational Geometry Algorithms Library (CGAL) is available at www.cgal.org.

${ }^{4}$ https://www.sofa-framework.org/
} 
in estimating myocardial deformations, especially the radial strain [14]. The sparse Demons algorithm was then selected to perform RV tacking given its good tracking accuracy and its low computational cost [43]. To avoid possible discontinuities at the LV/RV border, we refined the RV displacements by a simple method detailed in the appendix. The output of this procedure corresponds to a unique sequence of segmented meshes $\mathcal{R}_{t}$ attached to both cine and tagged MR.

\section{Template Warping}

As described in Section II-B4, a spatio-temporal alignment between the template sequences and each simulation frame is needed to derivate the proton density maps. We developed a novel warping framework to tackle this problem. Similar to [2], we used two different strategies to deal with the myocardium and the surrounding structures (named as background in the sequel), however we introduce the following innovative aspects: $i$ ) we developed a dedicated strategy to ensure smooth transition at the interface between the myocardium and the background; ii) we introduced a model to handle temporal myocardial intensity changes over the cardiac cycle. Each step of the proposed procedure is detailed hereunder.

1) Generic transformation model: Similar to [2], we used myocardial landmark points extracted from the two sequences of meshes $\mathcal{S}_{k}$ and $\mathcal{R}_{t}$ to compute displacement fields. In this study, we added static background landmarks (denoted as $\mathcal{B}$ ) to avoid non-physiological movement of the structures surrounding the heart muscle. These landmarks were detected from the cine MR template sequence. In particular, for each pixel position, we computed the variation of cine MR intensity over the whole sequence and we kept the points with the smallest variation and with a minimal spacing of $5 \mathrm{~mm}$. For each simulation, the number of static background landmarks was fixed to 200 .

The goal of the introduced generic model was to compute a global transformation to efficiently match a source space (represented by the heart mesh $\mathcal{M}_{0}$ ) with a target space (represented by the heart mesh $\mathcal{M}_{1}$ ) where $\mathcal{M}_{0}$ and $\mathcal{M}_{1}$ share the same number of points. In both spaces, we assumed the presence of the same static background landmarks $\mathcal{B}$. The transformation maps one pixel $\mathbf{x}$ of the source space to a corresponding position $\mathbf{y}$ in the target space. As stated above, different transformation strategies were applied to the myocardium and the background.

Regarding the myocardial points, since they all lie inside the volumetric mesh $\mathcal{M}_{0}$, the corresponding transformation was directly computed from the two volumetric meshes $\mathcal{M}_{0} \rightarrow \mathcal{M}_{1}$. The barycentric coordinates of $\mathrm{x}$ in mesh $\mathcal{M}_{0}$ were evaluated. From these barycentric coordinates and the node positions of the target mesh $\mathcal{M}_{1}$, we computed the absolute coordinates. This gave the matched position $\mathbf{y}$. This procedure is denoted as $\mathbf{y}=\operatorname{MESH} H_{\left\{\mathcal{M}_{0} \rightarrow \mathcal{M}_{1}\right\}}(\mathbf{x})$.

Concerning the background points, we used the Thin Plate Spline (TPS) algorithm to model the underlying transformation. We randomly selected a fraction of mesh points (800 points in all the simulations) which, together with the static

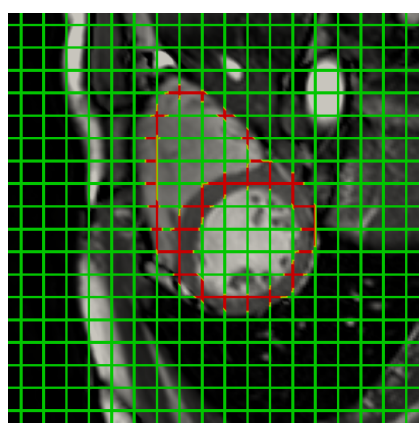

(a) initial cine MR image at ED

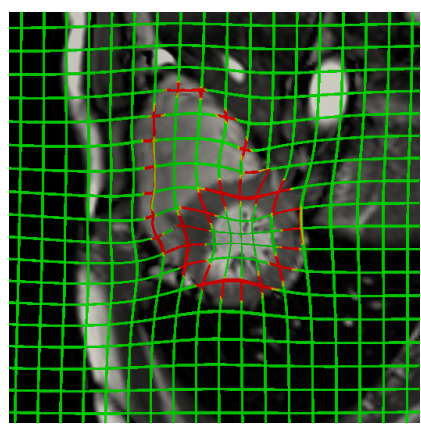

(b) image deformed to ES
Fig. 3: Illustration of the use of the generic transformation model. The ED cine MR image was warped to ES by the generic transformation. The grid superimposed on the image shows the corresponding deformation. The myocardium and the background are shown in red and green respectively. The generic transformation was computed from the ED/ES simulation meshes and the static background points.

landmarks, were used to parameterize the TPS algorithm. This procedure is denoted as $\mathbf{y}=T P S_{\left\{\mathcal{M}_{0}, \mathcal{B} \rightarrow \mathcal{M}_{1}, \mathcal{B}\right\}}(\mathbf{x})$. The overall transformation can thus be written as:

$$
\begin{aligned}
& \mathcal{T}_{\left\{\mathcal{M}_{0}, \mathcal{B} \rightarrow \mathcal{M}_{1}, \mathcal{B}\right\}}(\mathbf{x}) \\
& = \begin{cases}M E S H_{\left\{\mathcal{M}_{0} \rightarrow \mathcal{M}_{1}\right\}}(\mathbf{x}), & \text { if } \mathbf{x} \in \text { Myocardium } \\
\operatorname{TPS}_{\left\{\mathcal{M}_{0}, \mathcal{B} \rightarrow \mathcal{M}_{1}, \mathcal{B}\right\}}(\mathbf{x}), & \text { otherwise }\end{cases}
\end{aligned}
$$

Fig. 3 shows an example of the use of the generic transformation defined by Eq. 5 to derive a deformation map between two time instances.

2) Temporal alignment: Temporal alignment was performed by linearly stretching/shrinking the time axis of the template recording in order to match relevant cardiac events used as temporal landmarks in the simulation sequence. In this paper, ED and ES time events were chosen as landmarks. Each frame $k$ of the simulation was matched to a cardiac time $(\mathrm{ms})$ in the template sequence by the $\phi$ operator defined as:

$$
\phi(k)= \begin{cases}\frac{t^{e s}}{k^{e s}}, & \text { if } k \leq k^{e s} \\ t^{e s}+\frac{k-k^{e s}}{K-k^{e s}}\left(t^{\max }-t^{e s}\right), & \text { otherwise. }\end{cases}
$$

$K$ is the number of simulated frames and $k^{e s}$ corresponds to the ES frame index. Similarly, $t^{\max }$ is the template cardiac cycle while $t^{e s}$ refers to the template ES timing. Both the template cine and tagged MR sequences were resampled to cardiac timings $\phi(k)$, with $k \in[0, K-1]$. In each template sequence, the two frames with the cardiac times closest to $\phi(k)$ were found. These two images were then linearly interpolated to $\phi(k)$, producing template sequences temporally aligned with the simulation frames and denoted as $I_{\text {mod }}^{k}$.

3) Spatial alignment: After temporal synchronization, spatial alignment was needed to align the resampled images $I_{\text {mod }}^{k}$ with the corresponding E/M geometry $\mathcal{S}_{k}$. To this aim, the 
generic transformation introduced in Section II-D1 was used, yielding to the following expression:

$$
\hat{I}_{\text {mod }}^{k}=\dot{I}_{\text {mod }}^{k} \circ \mathcal{T}_{\left\{\mathcal{R}_{\phi(k)}, \mathcal{B} \rightarrow \mathcal{S}_{k}, \mathcal{B}\right\}}
$$

where $\hat{I}_{m o d}^{k}$ is the warped template image aligned both in time and space with the corresponding simulation mesh $\mathcal{S}_{k}$. The full spatio-temporal alignment strategy is given in Fig. 4.

4) Motion Correction: The warped template sequences $\hat{I}_{m o d}^{k}$ rely on motion tracking which may introduce artifacts in the synthetic images by adding an apparent residual motion to the true motion given by the E/M model. Contrary to [18], we introduced in this study a dedicated strategy that corrects the myocardial motion in the warped sequences. To ensure a coherence with the E/M model, the myocardial intensities of each frame of the simulated sequences were sampled from the first template images. This was achieved by deforming $I_{\text {mod }}^{0}$ to all simulation frames, yielding a complementary warped template sequence $\bar{I}_{\text {mod }}^{k}$ expressed as:

$$
\bar{I}_{\text {mod }}^{k}=I_{\text {mod }}^{0} \circ \mathcal{T}_{\left\{\mathcal{S}_{0}, \mathcal{B} \rightarrow \mathcal{S}_{k}, \mathcal{B}\right\}}
$$

This warped sequence was used to provide myocardial intensities while the warped sequence $\hat{I}_{\text {mod }}^{k}$ was used to get background intensities. In order to ensure a smooth interface between these two regions, we computed a Gaussian shaped weighting function centered at the myocardium for each simulation frame. Since most tracking algorithms aim to quantify the LV myocardium deformations, we only correct in this work the motion attached to this structure. For each simulation mesh $\mathcal{S}_{k}$ we extracted the LV surface and further converted it to an implicit function denoted as $\Gamma_{k}$. This function equals to 0 at the endo- and epicardium borders, is negative inside the myocardium and positive outside. Its minimal value is denoted as $\tau_{k}^{\min }$. The weighting function was then defined as follows:

$$
w_{k}(\mathbf{x})=\exp \left\{-\frac{\left(\Gamma_{k}(\mathbf{x})-\tau_{k}^{\min }\right)^{2}}{2 \sigma^{2}}\right\}
$$

where $\sigma$ controls the transmural profile of the weighting function and was set to 5 in all the simulations. The warped template sequences with myocardial motion correction were then computed as follows:

$$
\mathcal{I M}_{\text {mod }}^{k}=w_{k} \bar{I}_{\text {mod }}^{k}+\left(1-w_{k}\right) \hat{I}_{\text {mod }}^{k}
$$

Eq. (10) reveals that most of the myocardial intensities are assigned from the first template frame, ensuring coherent myocardial motion with the E/M model. The background intensities are principally retrieved from the spatiotemporally aligned template sequence, preserving the realistic nature of the surrounding structures.

5) Myocardial intensity changes: The warped template sequences $\mathcal{I M}_{\text {mod }}^{k}$ involved constant myocardium intensities over the cardiac cycle, which is too simplistic. We thus developed dedicated strategies for each modality to integrate myocardial temporal intensity changes in the simulated sequences.

Regarding tagged MR, Eq. (1) reveals that tagging intensity changes depend on $T_{1}$ relaxation and the RF flip angle. We thus used the static $T_{1}^{0}$ map together with the simulated RF flip angles $\alpha_{k}^{s}$ (details about its computation can be found in the appendix) to reproduce myocardial intensity variations for each simulated frame $k$ as follows:

$$
\Delta \mathcal{I} \mathcal{M}_{\text {tag }}^{k}=I_{\text {tag }}^{0}\left(\exp \left\{-\frac{k \Delta t^{s}}{T_{1}^{0}}\right\} \frac{\sin \left(\alpha_{k}^{s}\right)}{\sin \left(\alpha_{0}^{s}\right)} \prod_{j=0}^{k-1} \cos \left(\alpha_{j}^{s}\right)-1\right)
$$

where $\Delta t^{s}$ corresponds to the simulated temporal resolution, while $\Delta \mathcal{I} \mathcal{M}_{\text {tag }}^{k}$ corresponds to a non-warped sequence characterizing the temporal intensity changes at each pixel location.

Concerning cine MR, it can be observed that myocardial intensities increase from ED to ES and gradually decrease to their initial values at the end of the cardiac cycle. We assume that the intensity varies linearly during these two phases. The intensity change was defined as a percentage of the initial intensity. This percentage equals to 0 at $\mathrm{ED}, \xi$ at $\mathrm{ES}$ and changes linearly in between as follows:

$$
\lambda_{\xi}(t)= \begin{cases}\frac{t}{t^{e s}} \xi, & \text { if } t \leq t^{e s} \\ \frac{t^{\max }-t}{t^{\max }-t^{e s}} \xi, & \text { otherwise }\end{cases}
$$

where $t^{e s}$ and $t^{\max }$ were introduced in Section II-D2. The $\xi$ parameter was automatically computed from the template cine MR sequence for all myocardial points. In particular, for a given myocardial point $\mathbf{x}_{\mathbf{i}}$, we first retrieved the corresponding intensity values for all time instants thanks to the protocol described in Section II-C2. These intensities are denoted as $I_{\text {cine }}^{n}\left(\mathbf{x}_{i}\right)$ in the sequel, where $n$ corresponds to the cardiac cine MR frame instant. Each $\xi$ value related to a myocardial point $\mathbf{x}_{\mathbf{i}}$ was computed by fitting a linear model:

$$
\arg \min _{\xi} \sum_{n}\left(I_{\text {cine }}^{n}\left(\mathbf{x}_{i}\right)-\left(1+\lambda_{\xi}\left(t_{n}\right)\right) I_{\text {cine }}^{0}\left(\mathbf{x}_{i}\right)\right)^{2}
$$

Since Eq. (13) has a quadratic form with respect to $\xi$, its resolution is straightforward. For the points outside the myocardium, $\xi$ was set to 0 , meaning that their intensity does not vary over time. Finally, the intensity variations for each simulated frame $k$ was computed as:

$$
\Delta \mathcal{I} \mathcal{M}_{\text {cine }}^{k}=\lambda_{\xi}(\phi(k)) \cdot I_{\text {cine }}^{0}
$$

where $\phi(k)$ is the temporal alignment introduced in Section II-D2 and $I_{\text {cine }}^{0}$ is the first template cine MR image.

The myocardial intensity variations $\Delta \mathcal{I} \mathcal{M}_{m o d}^{k}$ were finally added to the previously generated warping images $\mathcal{I M}_{\text {mod }}^{k}$. Since the intensity variation maps were computed in static (i.e. without the integration of any motion), they had to be deformed before summation. The final warped template sequences with motion correction and integrating myocardial intensity changes were computed as follows:

$$
\tilde{I}_{\text {mod }}^{k}=\mathcal{I M}_{\text {mod }}^{k}+w_{k}\left(\Delta \mathcal{I} \mathcal{M}_{\text {mod }}^{k} \circ \mathcal{T}_{\left\{\mathcal{S}_{0}, \mathcal{B} \rightarrow \mathcal{S}_{k}, \mathcal{B}\right\}}\right)
$$

where $w_{k}$ is the weighting function given by Eq. (9). $\tilde{I}_{\text {cine }}^{k}$ and $\tilde{I}_{t a g}^{k}$ are therefore the final warped template cine/tagging sequences used to compute the proton density maps, as described in Section II-B4. 


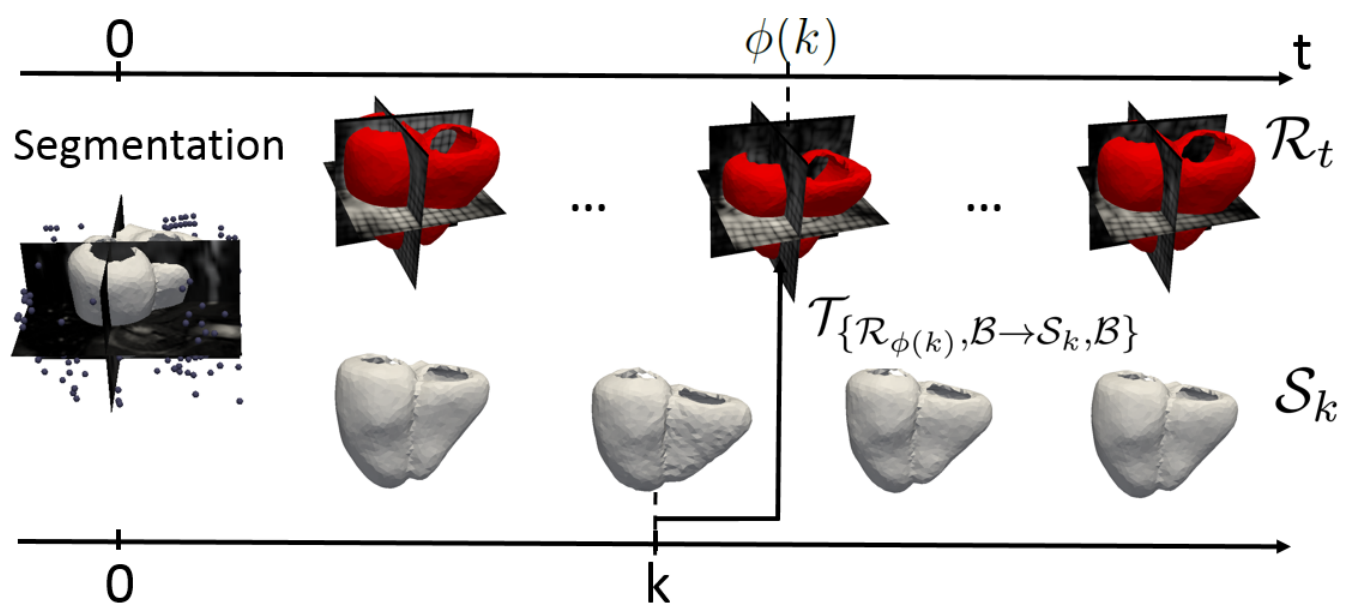

Fig. 4: Illustration of the temporal and spatial alignments described in Section II-D2 and II-D3 respectively. The myocardium is segmented at ED. The static background points are shown as black dots surrounding the mesh. The segmentation mesh is first tracked in the template sequence, yielding the tracking meshes $\mathcal{R}_{t}$ shown in red (the upper row). Then, the E/M model is exploited for simulating myocardial deformations starting from the same segmentation mesh, leading to another sequence of meshes $\mathcal{S}_{k}$ shown in the lower row. Each simulation frame $k$ is mapped to a cardiac time $\phi(k)$ of the template through the temporal alignment. The spatial alignment $\mathcal{T}_{\left\{\mathcal{R}_{\phi(k)}, \mathcal{B} \rightarrow \mathcal{S}_{k}, \mathcal{B}\right\}}$ then register the template image to the corresponding simulation mesh $\mathcal{S}_{k}$.

\section{E. US simulation}

As stated at the beginning of this Section, the same general pipeline (Fig. 1) was applied to simulate US sequences. Since the MR and US coordinate systems can easily be matched thanks to the manual landmarks described in Section II-A, the same initial mesh segmented from the first frame of the cine template was exploited. Only the "MR Modeling" block was replaced by the one proposed in [2] (block $\mathrm{C}$ in the paper). The underlying tasks allow the derivation of a 3D scatter map with backscattering amplitudes computed from the corresponding US template. From this map, an ultrasound volume was simulated by convolution with the spatially variant point spread function of the simulated imaging system. COLE [44] was adopted as ultrasound simulation environment due to its good compromise between computational efficiency and accuracy. The properties of the synthetic probe were set so to match as close as possible the ones of the transducer used in the acquisition of the template. More details related to the ultrasound simulation modeling can found in [2]. Contrary to [2], the derived US pipeline allows an explicit management of the transition between the myocardium and the surrounding structures, as further illustrated in Section IV.

\section{RESULT}

We used in this study three volunteers from [33]. For each volunteer, we simulated one healthy and five pathological cases (Section II-C1), yielding an open access database composed of 18 virtual patients. For each patient, five synthetic sequences were generated (one cine MR, three tagged MR and one US). All the simulations were launched from the virtual imaging platform VIP ${ }^{5}$ [19] which allows the execution of applications such as ODIN as a web service and benefits from

\footnotetext{
${ }^{5}$ https://vip.creatis.insa-lyon.fr
}

the $\mathrm{EGI}^{6}$ computing power. The generation of one full 3D sequence took around 6 hours on VIP, knowing that it would have taken over 280 hours $^{7}$ on a personal laptop.

\section{A. Image properties: qualitative assessment}

The proposed generic pipeline allows the simulation of multimodality volumes visually similar to real template recordings, as illustrated by Fig. 5 and 6 . The underlying global deformation values were found to be within clinical ranges (more details are given in Section III-C1). When considering the simulated cine loop, a realistic motion for the myocardium and the surrounding structures is observed (Fig. 5 and 6). For a better dynamical perception, more detailed images and movies of the full simulated sequences are provided in the supplementary materials (available in the supplementary files /multimedia tab) which constitute an essential support for an accurate evaluation of the method. Fig. 5 also reveals the efficiency of the Gaussian weighting function introduced in Section II-D4 to deal with the interface between the myocardium and the surrounding structures. Indeed, the proposed scheme allows a smooth transition between the different structures in this region while preserving a consistent motion inside the myocardium with the E/M model.

\section{B. Image properties: quantitative assessment}

Since the US simulation is based on the same scatter modeling as the one used in [2], the same image property is expected. We thus concentrate in this section on the MR image characteristics. In particular, the level of realism was assessed quantitatively by measuring the intensity distribution inside

\footnotetext{
${ }^{6}$ https://www.egi.eu

${ }^{7}$ estimation based on the time needed for computing one $2 \mathrm{D}$ slice on a personal computed with $16 \mathrm{G}$ of RAM and a $2.70 \mathrm{Ghz}$ CPU
} 


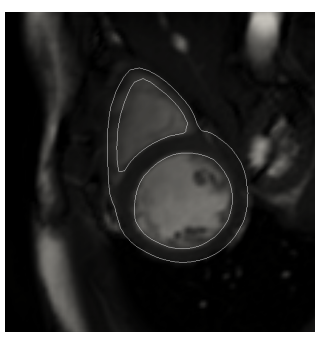

(a) c-MR at frame 0

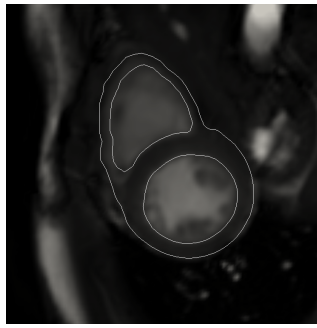

(f) c-MR at frame 5

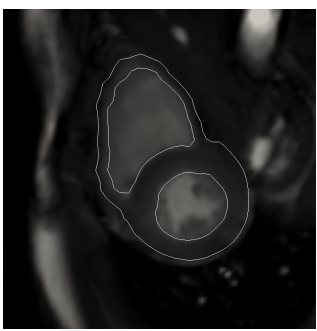

(k) c-MR at frame 10

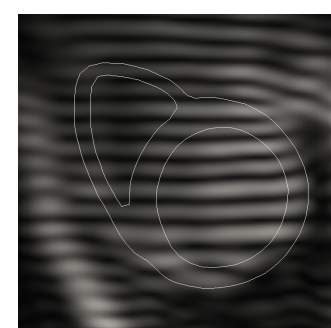

(b) t-MR (ch.1) at frame 0

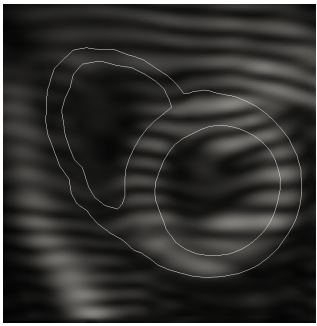

(g) t-MR (ch.1) at frame 5

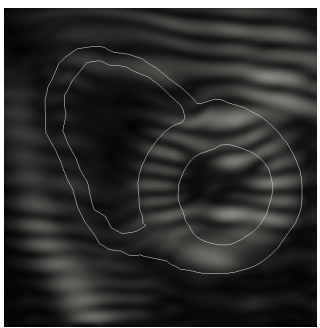

(1) t-MR (ch.1) at frame 10

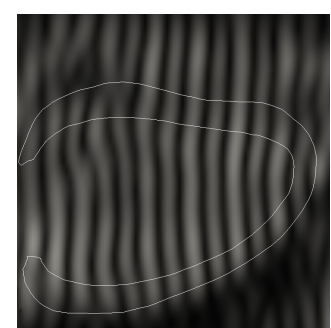

(c) t-MR (ch.2) at frame 0

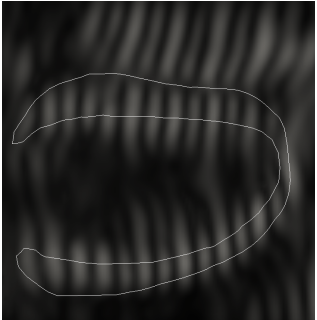

(h) t-MR (ch.2) at frame 5

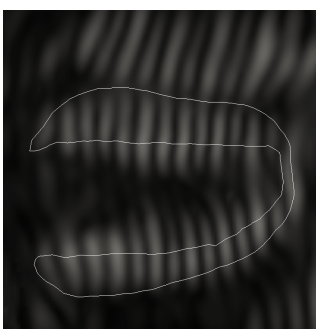

(m) t-MR (ch.2) at frame 10

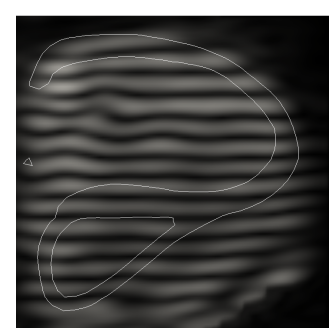

(d) t-MR (ch.3) at frame 0

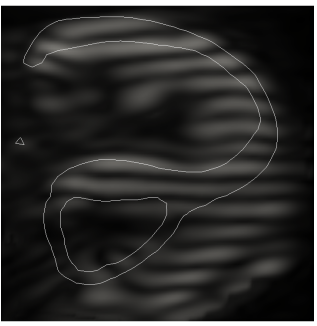

(i) t-MR (ch.3) at frame 5

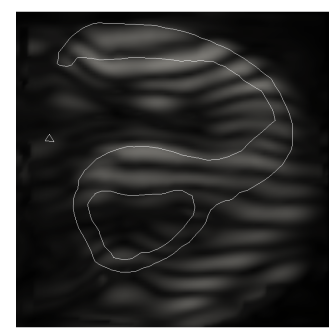

(n) t-MR (ch.3) at frame 10

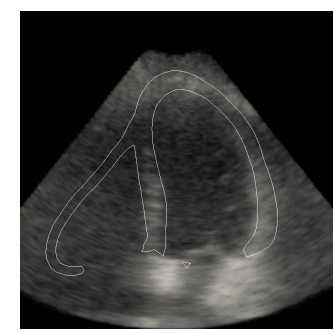

(e) US at frame 0

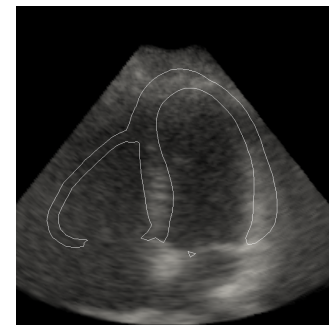

(j) US at frame 5

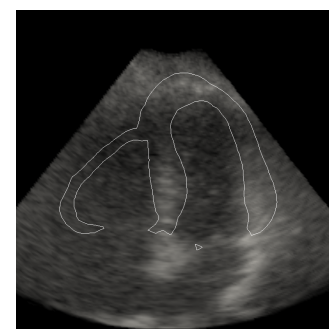

(o) US at frame 10

Fig. 5: Multimodality heart simulations from the same virtual patient at frame 0 (ED), frame 5 and frame 10 (ES). The E/M meshes (i.e. ground truth segmentation) are superimposed as white contours. Videos showing the entire simulated sequences are provided in the supplementary materials (available in the supplementary files /multimedia tab).

the myocardium since it is the feature commonly exploited for the analysis of MR cardiac datasets. The myocardial histograms at end diastole (ED) and end systole (ES) for both a simulated cine MR sequence and its corresponding template are reported in Fig. 7. The analysis first demonstrated that the computed histograms showed very similar shapes at ED. This is consistent with the fact that ED corresponds to the reference time in the proposed pipeline from which $\mathrm{T} 1, \mathrm{~T} 2$ and proton density maps were directly computed from the corresponding template frame. This result is further confirmed by two statistical tests. Both the Wilcoxon signedrank test and the Levene's test returned p-value greater than 0.05 , meaning that the median and variance of the myocardial intensities are statistically similar. At ES, the histograms are still in a good agreement, even if some slight difference may be observed. We applied the same statistic tests on the myocardial intensities. The Wilcoxon signed-rank test returned a p-value greater than 0.05 , revealing that the template and the simulation have similar median values. However, the Levene's test returned a p-value below 0.05 , meaning that the variances are statistically different. This is coherent with what we see from the histograms. This is due to the fact that intensities of the T1 and T2 maps were not updated over the cardiac cycle, only warping strategies were performed. Moreover, the myocardial intensity changes were embedded through the proton density maps which was modeled through a simple linear fit. Nevertheless, the similarity of the distributions validates the proposed strategy to handle the myocardial intensity changes while preserving the motion derived from the $\mathrm{E} / \mathrm{M}$ model. The myocardial histograms of tagged MR simulations are provided in the supplementary materials (available in the supplementary files /multimedia tab). Results show that the exact same observations can also be made for this modality.

\section{Mechanical properties}

1) Global indices: Table II displays the LV volumes at ED and ES along with the corresponding EF and the LV mass for the healthy and pathological cases. Those values were computed from the simulated meshes $\mathcal{S}_{k}$ used as benchmark for the proposed database. For the healthy case, the LV volumes present values around $104 \mathrm{~mL}$ at $\mathrm{ED}$ and $50 \mathrm{~mL}$ at ES, which is in agreement with the reference range published in [45] when combining all ethnic groups, all ages and both sexes in the analysis. In the case of dyssynchrony, the ES volumes slightly increase to values around $53 \mathrm{~mL}$, revealing a reduced blood output. This tendency is clearer for ischemic 


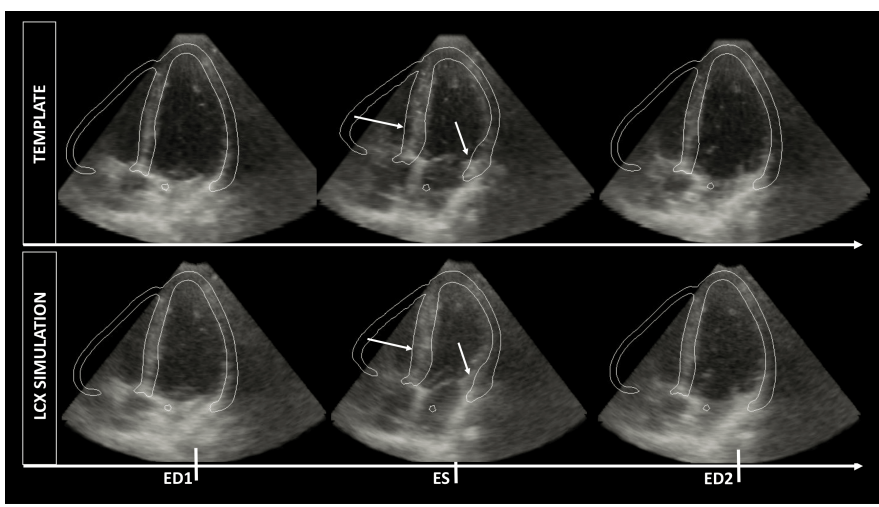

(a) US template recording and LCX simulation for an apical four-chamber slice

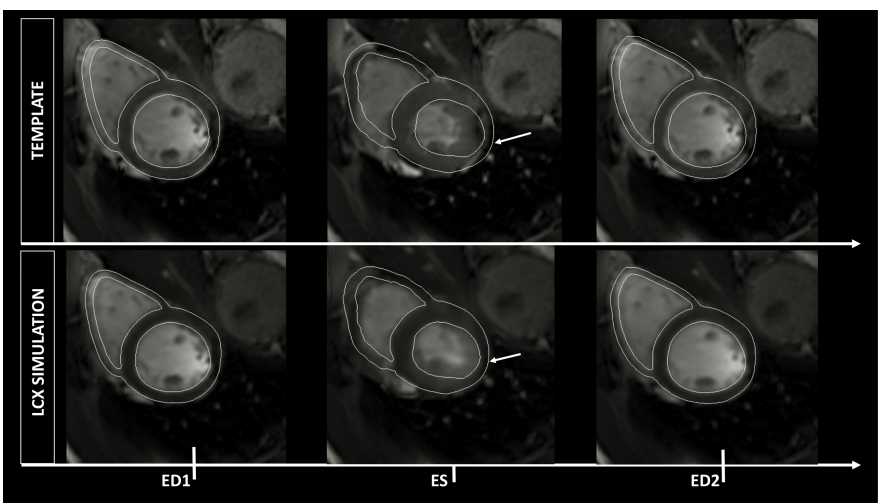

(c) cine MR template recording and LCX simulation for a short-axis slice

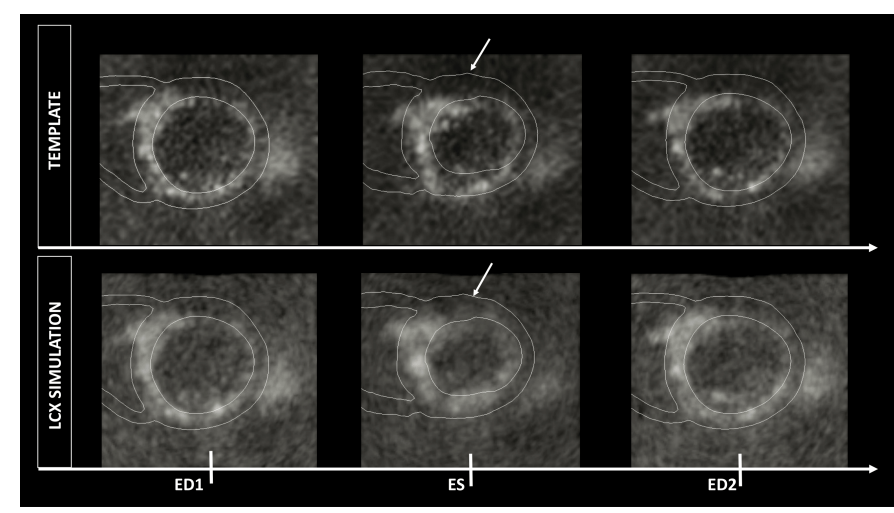

(b) US template recording and LCX simulation for a short-axis slice

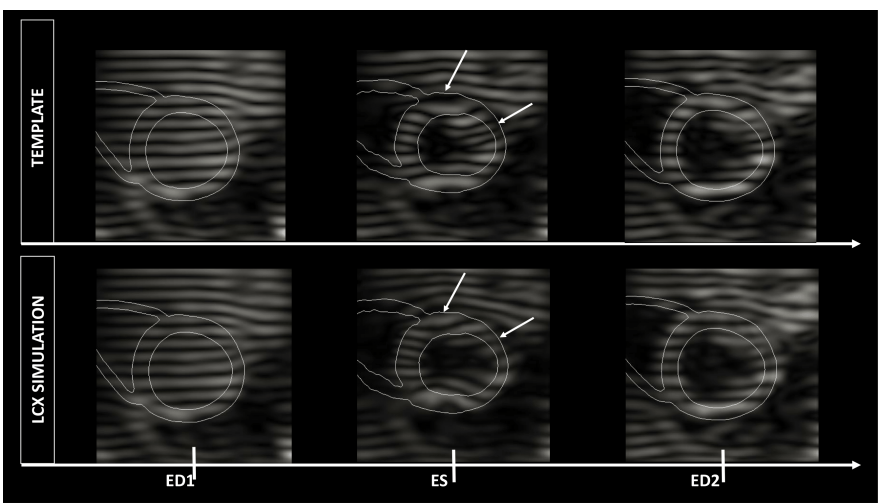

(d) tagged MR template recording and LCX simulation for a short-axis slice

Fig. 6: Template US and MR recordings (top row) and LCX simulation (bottom row) at matched time instants for a fourchamber US slice (a), a short-axis US slice (b), a short-axis cine MR slice (c) and a short-axis tagged MR slice (d). The E/M meshes (i.e. ground truth segmentation of the LCX simulation) are superimposed to each sequence as white contours. Template, simulation and, therefore, E/M geometry are aligned by construction at time zero (i.e. at ED). From then on, the simulation runs independently of the template recording and, therefore, the E/M geometry no longer provides a valid segmentation of the latter. In the figure, this is particularly visible at regions marked by the white arrows. This is a consequence of the fact that, due to the simulated ischemia, contraction in the $\mathrm{E} / \mathrm{M}$ simulation is reduced when compared to the template.

cases where ES volumes are above $60 \mathrm{~mL}$. Regarding the EF, the values are around $52 \%$ for healthy cases and concentrated around $40 \%$ for ischemic cases. The EF of the healthy cases are rather close to but slightly below the reference values from the literature (above 60\%) [46]. Concerning the LV mass, the values corresponding to the three healthy cases (median value around $110 \mathrm{~g}$ ) are all within the reference range reported in [45] and [47].

2) Strain Curves: We show in Fig. 8 the full set of regional strain (radial, longitudinal and circumferential) curves at the basal, mid and apical levels of the LV for one healthy and four pathological cases. For the ischemic cases, we also provided in the supplementary materials (available in the supplementary files /multimedia tab) the ground truth infarction areas projected onto the AHA segments. The regional strain curves were computed using Engineering strain as in [2]. Their properties are in accordance with the ones described in the literature. For instance, in the LBBB case, the ES timings differ between the septum (AHA n 2,3,8,9,14) and the lateral wall (AHA n $5,6,11,12,16)$. The septum is activated slightly before the lat- eral wall, meaning the myocardium contracts asynchronously. Besides, ischemic cases show wide disparities between healthy and pathological segments. Reduced deformation values are observed for those infarcted AHA segments.

In addition, we show in Fig. 9 the global circumferential strain (GCS) for the 18 virtual patients simulated in this paper. We observe that pathological cases display reduced circumferential deformations compared to healthy ones.

\section{Discussion}

We introduced in this paper a generic framework allowing the generation of realistic multimodal (US, cine and tagged MR) cardiac synthetic sequences for the same virtual patient. Based on our previous work described in [2], the main novelties of the proposed technique are two fold: i) we extended the pipeline originally developed for US to MR simulation; ii) we developed a dedicated scheme to better handle the transition between the myocardium and the surrounding structures.

It is widely acknowledged that multimodality raises many issues in cardiac imaging, beyond the mapping of all in- 


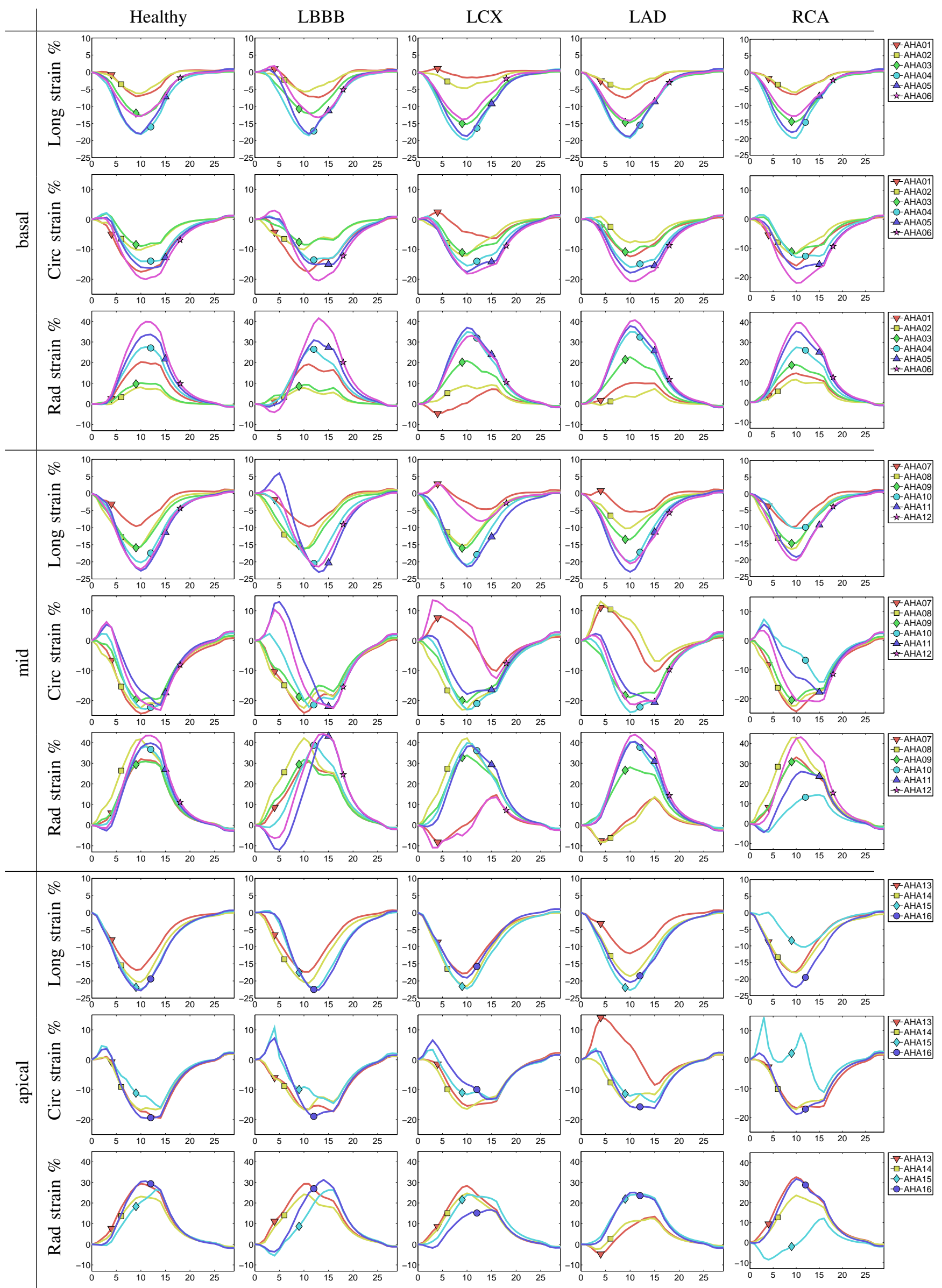

Fig. 8: Regional strain curves at basal, mid and apical levels of the LV. One healthy, one dynssynchrony (LBBB) and three infarcted hearts (LCX, LAD, RCA) are shown. The colormap used for displaying the curves is provided in the appendix. 

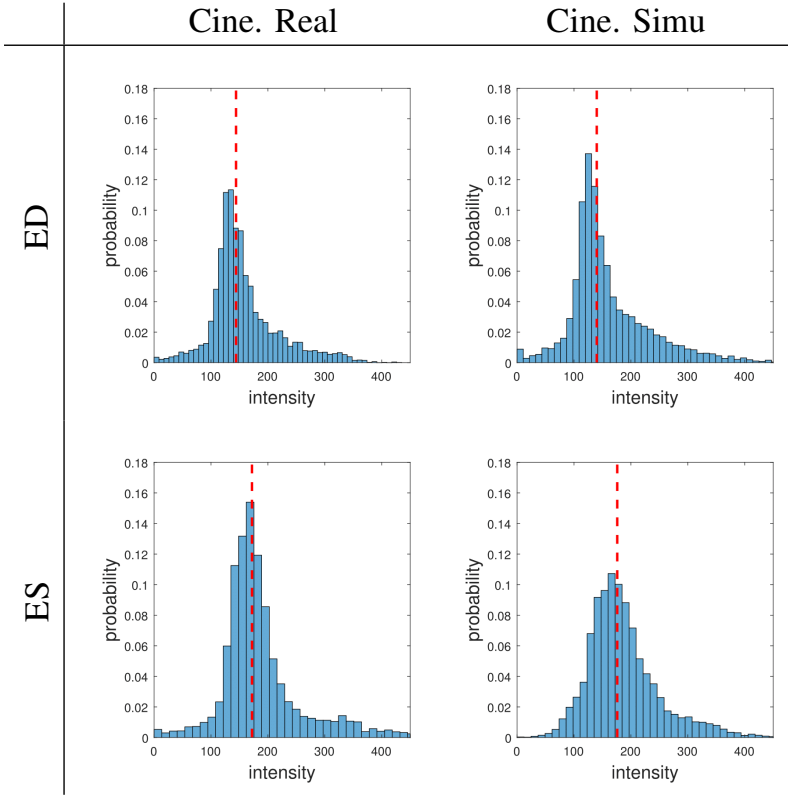

Fig. 7: Histograms of myocardial intensities in both real and simulated MR data. Red dashed lines mark median intensities.

formation in a common reference space. Indeed, different modalities were reported to provide significant differences for the quantification of volumes and ejection fraction values [48]. Offering to the community a consolidated dataset, on the basis of which generic or modality-specific algorithms can be quantitatively compared, is a step forward towards understanding whether reported differences between modalities originate from the processing, the difference in image quality or can only be explained by more fundamental differences in the physics of imaging the same complex tissue though

TABLE II: LV volumes at ED/ES, the corresponding EF and the LV mass distributed among the virtual population.

Patient 1

\begin{tabular}{ccccccc}
\hline & Healthy & LBBB & LCX & LAD & RCA & RCA2 \\
\hline EDV $(\mathrm{mL})$ & 106.7 & 106.7 & 106.7 & 106.7 & 106.7 & 106.7 \\
ESV (mL) & 45.7 & 48.8 & 64.6 & 57.1 & 57.8 & 59.1 \\
EF $(\%)$ & 57.2 & 54.3 & 39.5 & 46.5 & 45.8 & 44.6 \\
LV mass $(\mathrm{g})$ & 116.8 & 116.8 & 116.8 & 116.8 & 116.8 & 116.8 \\
\hline
\end{tabular}

Patient 2

\begin{tabular}{ccccccc}
\hline & Healthy & LBBB & LCX & LAD & RCA & RCA2 \\
\hline EDV $(\mathrm{mL})$ & 107.6 & 107.6 & 107.6 & 107.6 & 107.6 & 107.6 \\
ESV $(\mathrm{mL})$ & 50.5 & 53.9 & 61.4 & 59.7 & 60.7 & 63.3 \\
EF $(\%)$ & 53.1 & 49.9 & 42.9 & 44.5 & 43.6 & 41.2 \\
LV mass $(\mathrm{g})$ & 125.1 & 125.1 & 125.1 & 125.1 & 125.1 & 125.1 \\
\hline
\end{tabular}

Patient 3

\begin{tabular}{ccccccc}
\hline & Healthy & LBBB & LCX & LAD & RCA & RCA2 \\
\hline EDV (mL) & 97.8 & 97.8 & 97.8 & 97.8 & 97.8 & 97.8 \\
ESV (mL) & 52.5 & 55.8 & 71.1 & 65.8 & 62.5 & 67.7 \\
EF $(\%)$ & 46.3 & 42.9 & 27.3 & 32.7 & 36.1 & 30.8 \\
LV mass $(\mathrm{g})$ & 100.0 & 100.0 & 100.0 & 100.0 & 100.0 & 100.0 \\
\hline
\end{tabular}

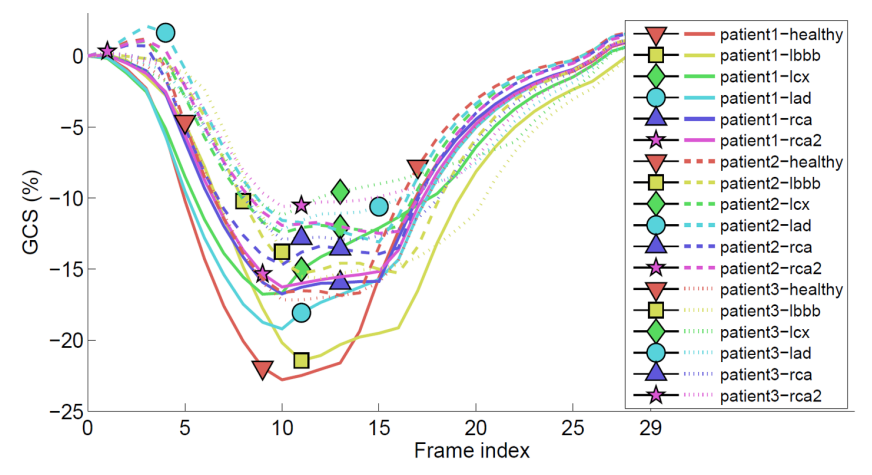

Fig. 9: Global circumferential strain curves corresponding to the 18 simulated virtual patients.

different modalities. By construction, our simulation method ensures that the synthetic MR and US sequences are generated from the same patient. The E/M simulations generated in the MR system of coordinates were brought to the US system by a simple rigid transformation composed of translations and rotations. The interest of such rigid transformation resides in the fact that it will not introduce any local myocardium deformation. The deformation patterns represented in the US and MR sequences are therefore the same, allowing a straightforward cross-modality comparison. However, this rigid transformation is sensitive to the accuracy of the 24 manual landmarks introduced in Section II-A. The manual landmarking should thus be carefully performed in order to minimize the potential impact on the US simulations.

By combining warpings of the whole template sequence and of the first frame only to each simulation mesh, we obtained a realistic background while guaranteeing that no registration error can introduce artifacts inside the myocardium. This is a fundamental difference with all registration-based methods such as [18] where the error on displacements gets composed with the true displacement field, making any derived benchmark affected by such artifacts inside the myocardium. Moreover, we introduced an efficient Gaussian weighting function to deal with the interface between the myocardium and the background. Such a scheme guarantees a smooth transition between the different structures around the myocardium and thus avoids potential artifacts as it may appear for instance from the pipeline developed in [2] (Fig. 10).

Despite the advantages, we also acknowledge a number of limitations and simplifications. First, the realism of the computed relaxation times $T_{1}$ and $T_{2}$ could be improved to account for the temporal variations of these quantities as reported in [49]. The corresponding spatial distribution we used was kept simple (average reference values + Gaussian noise for the background region). Also, the myocardial $T_{1}$ values we computed from real tagging templates should be compared against real $T_{1}$ mapping acquisitions to validate this process. Additionally, when computing the proton density for tagged MR simulation, we assumed the effective spinspin relaxation time $T_{2}^{*}$ to equal the theoretical $T_{2}$ value. This assumption simplifies the simulation method and since they have close values, it should not significantly impact the 


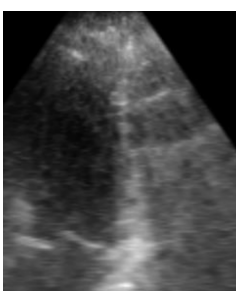

(a) Template image

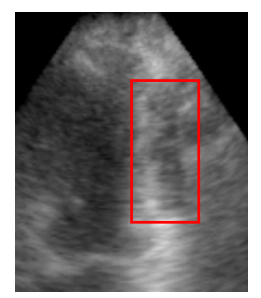

(b) result obtained using [2]

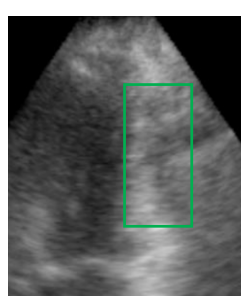

(c) result derived from our method
Fig. 10: Illustration of the interest of the proposed method to better handle with the interface between myocardium and the surrounding structures. a) template image at ES; b) simulated image using [2]; c) simulated image using our approach.

simulation results. It should be emphasized however that our modeling strategy was aiming at optimizing the realism of the generated images rather than rigorously following the underlying physics. With this in mind, the derivations of most MR parameters we made from real MR images ensure a high level of realism of the simulated images as shown in Fig. 5 and 6.

We put a lot of effort in this paper to make sure that tracking errors would not introduce any artifacts inside the myocardium. However, it is worth mentioning that the quality of the simulated data remains sensitive to the tracking accuracy. Since the background is taken from a frame of the template sequence, tracking errors will appear as a residual motion applied to the background, resulting in possible tag line fractures occurring at the myocardium/background interface, although the use of a Gaussian weighting function is expected to limit such unrealistic artifacts. An accurate tracking is therefore required for producing high quality simulation data, which we ensured by using high quality images from carefully selected healthy subjects [33].

Finally, the median longitudinal strain corresponding to the cardiac deformations generated by the E/M model is around $15 \%$, which is still lower than reference clinical values. This has already been discussed in [2] and remains one of the major limitations of the E/M model. Compared to [2], the simulations generated in this paper already show increased longitudinal deformations thanks to a thorough parameter tuning process. Further improvement of the longitudinal strain as well as the reduction of the dispersion of GCS and the increase of the EF remain the object of future work.

\section{CONCLUSION}

In this paper, we developed a generic pipeline for simulating 3D synthetic cardiac cine MR, tagged MR and US image sequences. The groundtruth cardiac motion fields were generated by an electro-mechanical model. Template acquisitions were deformed into the simulation space by a novel warping technique which ensures that i) motion in the synthetic images matches the E/M model and ii) transitions at the myocardium/background interface are smooth. Both MR and US images were generated by relevant physical simulators. We analyzed the image properties both qualitatively and quantitatively. In total, we simulated 18 virtual subjects representing 90 synthetic sequences which are made publicly available via an open-access database. We plan in a future work to use this synthetic database to conduct a comprehensive and thorough validation study. In particular, performances of state-of-the-art motion tracking algorithms will be compared across different modalities.

\section{AKNOWLEDGMENT}

The research leading to these results has received funding from European Union through the Seventh Framework Programme (FP7/2007-2013) under grant agreement VP2HF No. 611823 and the Horizon 2020 Programme for Research and Innovation, under grant agreement CardioFunXion No. 642676.

\section{APPENDIX}

\section{A. Refine the tracking of $R V$}

In Sect. II-C2, LV and RV were tracked separately. To avoid possible distortions at the LV/RV border, we propose to update RV displacements by a method detailed in the following. We first built a Thin plate spline transformation $\Psi$ upon LV displacements tracked by HarpAR. The displacements of RV points $\mathrm{x}$ are then corrected a posteriori by weighted sums of $\Psi(\mathbf{x})$ and SparseDemons tracking results denoted as $\mathbf{u}^{s}(\mathbf{x})$ :

$$
\begin{aligned}
& \mathbf{u}(\mathbf{x})=v(\mathbf{x}) \Psi(\mathbf{x})+(1-v(\mathbf{x})) \mathbf{u}^{s}(\mathbf{x}) \\
& \text { with } \quad v(\mathbf{x})=e^{-\frac{d(\mathbf{x})}{r}}
\end{aligned}
$$

where $d(\mathbf{x})$ is the distance of $\mathbf{x}$ to the LV. $r$ is a constant with default value equals $5 \mathrm{~mm}$ in our experiments.

\section{B. Compute RF flip angles}

In Sect. II-B2, we mentioned that RF flip angles could be easily computed. We explain the details in the following part. In CSPAMM acquisitions [38], RF flip angles are ensured to satisfy the following condition [50]:

$$
\alpha_{n-1}=\arctan \left(\sin \left(\alpha_{n}\right)\right) \cdot \exp \left\{-\frac{\Delta t}{T_{1}^{\text {myo }}}\right\}
$$

where $\alpha_{n}$ is the flip angle for acquiring the frame $n, \Delta t$ is the temporal resolution and $T_{1}^{\text {myo }}$ is the average myocardial $T_{1}$ relaxation time. Since the last RF flip angle was available from the DICOM header, we then derived all the RF flip angles backward by Eq. 17. Similarly, Eq. 17 was used for computing all the flip angles of the simulations denoted as $\alpha_{k}^{s}$.

\section{REFERENCES}

[1] S. Mendis, Global status report on noncommunicable diseases 2014. World Health Organization reports, 2014.

[2] M. Alessandrini, M. De Craene, O. Bernard, S. Giffard-Roisin, P. Allain, I. Waechter-Stehle, J. Weese, E. Saloux, H. Delingette, M. Sermesant et al., "A pipeline for the generation of realistic $3 \mathrm{~d}$ synthetic echocardiographic sequences: methodology and open-access database," IEEE transactions on medical imaging, vol. 34, no. 7, pp. 1436-1451, 2015.

[3] L. Axel, A. Montillo, and D. Kim, "Tagged magnetic resonance imaging of the heart: a survey," Medical image analysis, vol. 9, no. 4, pp. 376393, 2005. 


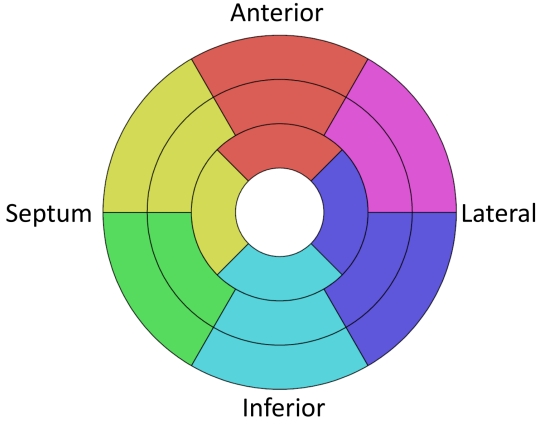

Fig. 11: The colormap used for the display of regional strain curves.

[4] H. Wang and A. A. Amini, "Cardiac motion and deformation recovery from mri: a review," IEEE Transactions on Medical Imaging, vol. 31, no. 2, pp. 487-503, 2012.

[5] W. Shi, X. Zhuang, H. Wang, S. Duckett, D. V. Luong, C. TobonGomez, K. Tung, P. J. Edwards, K. S. Rhode, R. S. Razavi et al., "A comprehensive cardiac motion estimation framework using both untagged and 3-d tagged mr images based on nonrigid registration," IEEE transactions on medical imaging, vol. 31, no. 6, pp. 1263-1275, 2012.

[6] O. A. Smiseth, H. Torp, A. Opdahl, K. H. Haugaa, and S. Urheim, "Myocardial strain imaging: how useful is it in clinical decision making?" European heart journal, p. ehv529, 2015.

[7] H. Blessberger and T. Binder, "Two dimensional speckle tracking echocardiography: basic principles," Heart, vol. 96, no. 9, pp. 716-722, 2010.

[8] S. Cheng, M. G. Larson, E. L. McCabe, E. Osypiuk, B. T. Lehman, P. Stanchev, J. Aragam, E. J. Benjamin, S. D. Solomon, and R. S Vasan, "Reproducibility of speckle-tracking-based strain measures of left ventricular function in a community-based study," Journal of the American Society of Echocardiography, vol. 26, no. 11, pp. 1258-1266, 2013.

[9] K. Shiino, A. Yamada, M. Ischenko, B. K. Khandheria, M. Hudaverdi, V. Speranza, M. Harten, A. Benjamin, C. R. Hamilton-Craig, D. G. Platts et al., "Intervendor consistency and reproducibility of left ventricular $2 \mathrm{~d}$ global and regional strain with two different high-end ultrasound systems," Eur Heart J Cardiovasc Imaging, p. jew120, 2016.

[10] S. A. Kleijn, M. F. Aly, C. B. Terwee, A. C. van Rossum, and O. Kamp, "Reliability of left ventricular volumes and function measurements using three-dimensional speckle tracking echocardiography," European Heart Journal-Cardiovascular Imaging, vol. 13, no. 2, pp. 159-168, 2012.

[11] G. K. Singh, B. Cupps, M. Pasque, P. K. Woodard, M. R. Holland, and A. Ludomirsky, "Accuracy and reproducibility of strain by speckle tracking in pediatric subjects with normal heart and single ventricular physiology: a two-dimensional speckle-tracking echocardiography and magnetic resonance imaging correlative study," Journal of the American Society of Echocardiography, vol. 23, no. 11, pp. 1143-1152, 2010.

[12] G. Pedrizzetti, P. Claus, P. J. Kilner, and E. Nagel, "Principles of cardiovascular magnetic resonance feature tracking and echocardiographic speckle tracking for informed clinical use," Journal of Cardiovascular Magnetic Resonance, vol. 18, no. 1, p. 51, 2016.

[13] M. Alessandrini, B. Heyde, S. Queirós, S. Cygan, M. Zontak, O. Somphone, O. Bernard et al., "Detailed evaluation of five 3d speckle tracking algorithms using synthetic echocardiographic recordings," IEEE Transactions on Medical Imaging, vol. 35, no. 8, pp. 1915-1926, 2016.

[14] Y. Zhou, O. Bernard, E. Saloux, A. Manrique, P. Allain, S. MakramEbeid, and M. De Craene, "3d harmonic phase tracking with anatomical regularization,” Medical Image Analysis, vol. 26, no. 1, pp. 70-81, 2015.

[15] K. N. Hor, R. Baumann, G. Pedrizzetti, G. Tonti, W. M. Gottliebson, M. Taylor, D. W. Benson, and W. Mazur, "Magnetic resonance derived myocardial strain assessment using feature tracking," JoVE (Journal of Visualized Experiments), no. 48, pp. e2356-e2356, 2011.

[16] W. E. Moody, R. J. Taylor, N. C. Edwards, C. D. Chue, F. Umar, T. J. Taylor, C. J. Ferro, A. A. Young, J. N. Townend, F. Leyva et al., "Comparison of magnetic resonance feature tracking for systolic and diastolic strain and strain rate calculation with spatial modulation of magnetization imaging analysis," Journal of Magnetic Resonance Imaging, vol. 41, no. 4, pp. 1000-1012, 2015.
[17] T. Onishi, S. K. Saha, D. R. Ludwig, T. Onishi, J. J. Marek, J. L. Cavalcante, E. B. Schelbert, D. Schwartzman, and J. Gorcsan, "Feature tracking measurement of dyssynchrony from cardiovascular magnetic resonance cine acquisitions: comparison with echocardiographic speckle tracking," Journal of Cardiovascular Magnetic Resonance, vol. 15, no. 1, p. $95,2013$.

[18] A. Prakosa, M. Sermesant, H. Delingette, S. Marchesseau, E. Saloux, P. Allain, N. Villain, and N. Ayache, "Generation of synthetic but visually realistic time series of cardiac images combining a biophysical model and clinical images," Medical Imaging, IEEE Transactions on, vol. 32, no. 1, pp. 99-109, 2013.

[19] T. Glatard, C. Lartizien, B. Gibaud, R. F. Da Silva, G. Forestier, F. Cervenansky, M. Alessandrini, H. Benoit-Cattin, O. Bernard, S. CamarasuPop et al., "A virtual imaging platform for multi-modality medical image simulation," IEEE Transactions on Medical Imaging, vol. 32, no. 1, pp. 110-118, 2013.

[20] P. Clarysse Sr, J. Tafazzoli, P. Delachartre, and P. Croisille, "Simulation based evaluation of cardiac motion estimation methods in tagged-mr image sequences," Journal of Cardiovascular Magnetic Resonance, vol. 13, no. 1, pp. 1-1, 2011.

[21] Y. Zhou, M. De Craene, O. Somphone, M. Sermesant, and O. Bernard, "Generation of realistic 4d synthetic cspamm tagged mr sequences for benchmarking cardiac motion tracking algorithms," in International Workshop on Simulation and Synthesis in Medical Imaging. Springer, in press, pp. 212-219.

[22] C. Tobon-Gomez, F. Sukno, B. Bijnens, M. Huguet, and A. Frangi, "Realistic simulation of cardiac magnetic resonance studies modeling anatomical variability, trabeculae, and papillary muscles," Magnetic Resonance in Medicine, vol. 65, no. 1, pp. 280-288, 2011.

[23] L. Wissmann, C. Santelli, W. P. Segars, and S. Kozerke, "Mrxcat: Realistic numerical phantoms for cardiovascular magnetic resonance," Journal of Cardiovascular Magnetic Resonance, vol. 16, no. 1, p. 1 , 2014.

[24] W. Segars, G. Sturgeon, S. Mendonca, J. Grimes, and B. M. Tsui, " $4 d$ xcat phantom for multimodality imaging research," Medical physics, vol. 37, no. 9, pp. 4902-4915, 2010.

[25] R. Haddad, I. E. Magnin, and P. Clarysse, "A new fully-digital anthropomorphic and dynamic thorax/heart model," in Engineering in Medicine and Biology Society, 2007. EMBS 2007. 29th Annual International Conference of the IEEE. IEEE, 2007, pp. 5999-6002.

[26] R. Haddad, P. Clarysse, M. Orkisz, P. Croisille, D. Revel, and I. E. Magnin, "A realistic anthropomorphic numerical model of the beating heart," in International Workshop on Functional Imaging and Modeling of the Heart. Springer, 2005, pp. 384-393.

[27] W. R. Crum, E. Berry, J. P. Ridgway, U. M. Sivananthan, L.-B. Tan, and M. A. Smith, "Simulation of two-dimensional tagged mri," Journal of Magnetic Resonance Imaging, vol. 7, no. 2, pp. 416-424, 1997.

[28] W. R. Crum, E. Berry, J. P. Ridgway, U. M. Sivananthan, L. B. Tan, and M. A. Smith, "Frequency-domain simulation of mr tagging," Journal of Magnetic Resonance Imaging, vol. 8, no. 5, pp. 1040-1050, 1998.

[29] E. Waks, J. L. Prince, and A. S. Douglas, "Cardiac motion simulator for tagged mri," in Mathematical Methods in Biomedical Image Analysis, 1996., Proceedings of the Workshop on. IEEE, 1996, pp. 182-191.

[30] M. Sermesant, P. Moireau, O. Camara, J. Sainte-Marie, R. Andriantsimiavona, R. Cimrman, D. L. Hill, D. Chapelle, and R. Razavi, "Cardiac function estimation from mri using a heart model and data assimilation: advances and difficulties," Medical Image Analysis, vol. 10, no. 4, pp. 642-656, 2006.

[31] M. De Craene, S. Marchesseau, B. Heyde, H. Gao, M. Alessandrini, O. Bernard, G. Piella, A. Porras, L. Tautz, A. Hennemuth et al., "3d strain assessment in ultrasound (straus): A synthetic comparison of five tracking methodologies," IEEE transactions on medical imaging, vol. 32, no. 9, pp. 1632-1646, 2013.

[32] S. Marchesseau, H. Delingette, M. Sermesant, and N. Ayache, "Fast parameter calibration of a cardiac electromechanical model from medical images based on the unscented transform," Biomechanics and modeling in mechanobiology, vol. 12, no. 4, pp. 815-831, 2013.

[33] C. Tobon-Gomez, M. De Craene, K. Mcleod, L. Tautz, W. Shi, A. Hennemuth, A. Prakosa, H. Wang, G. Carr-White, S. Kapetanakis et al., "Benchmarking framework for myocardial tracking and deformation algorithms: An open access database," Medical Image Analysis, vol. 17, no. 6, pp. 632-648, 2013.

[34] K. Scheffler and S. Lehnhardt, "Principles and applications of balanced ssfp techniques," European radiology, vol. 13, no. 11, pp. 2409-2418, 2003. 
[35] A. K. Rutz, S. Ryf, S. Plein, P. Boesiger, and S. Kozerke, "Accelerated whole-heart 3d cspamm for myocardial motion quantification," Magnetic Resonance in Medicine, vol. 59, no. 4, pp. 755-763, 2008.

[36] T. H. Jochimsen, A. Schäfer, R. Bammer, and M. E. Moseley, "Efficient simulation of magnetic resonance imaging with bloch-torrey equations using intra-voxel magnetization gradients," Journal of Magnetic Resonance, vol. 180, no. 1, pp. 29-38, 2006.

[37] A. Groth, J. Weese, and H. Lehmann, "Robust left ventricular myocardium segmentation for multi-protocol mr," in SPIE Medical Imaging. International Society for Optics and Photonics, 2012.

[38] S. E. Fischer, G. McKinnon, S. Maier, and P. Boesiger, "Improved myocardial tagging contrast," Magnetic resonance in medicine, vol. 30, no. 2, pp. 191-200, 1993.

[39] H. Wang, M. Kadbi, M. Kotys, M. Ersoy, G. P. Chatzimavroudis, R. M. Setser, M. Alshaher, S. E. Fischer, and A. A. Amini, "Orthogonal cspamm (ocspamm) $\mathrm{mr}$ tagging for imaging ventricular wall motion," in 2011 Annual International Conference of the IEEE Engineering in Medicine and Biology Society. IEEE, 2011, pp. 535-538.

[40] A. Haase, "Snapshot flash mri. applications to t1, t2, and chemical-shift imaging," Magnetic Resonance in Medicine, vol. 13, no. 1, pp. 77-89, 1990.

[41] The CGAL Project, CGAL User and Reference Manual, 4.9 ed. CGAL Editorial Board, 2016. [Online]. Available: http://doc.cgal.org/4. 9/Manual/packages.html

[42] N. Duchateau, M. De Craene, P. Allain, E. Saloux, and M. Sermesant, "Infarct localization from myocardial deformation: Prediction and uncertainty quantification by regression from a low-dimensional space." IEEE transactions on medical imaging, 2016.

[43] O. Somphone, M. De Craene, R. Ardon, B. Mory, P. Allain, H. Gao, J. D'hooge, S. Marchesseau, M. Sermesant, H. Delingette et al., "Fast myocardial motion and strain estimation in $3 \mathrm{~d}$ cardiac ultrasound with sparse demons," in Biomedical Imaging (ISBI), 2013 IEEE 10th International Symposium on. IEEE, 2013, pp. 1182-1185.

[44] H. Gao, H. F. Choi, P. Claus, S. Boonen, S. Jaecques, G. H. V. Lenthe, G. V. D. Perre, W. Lauriks, and J. D'hooge, "A fast convolution-based methodology to simulate 2-d/3-d cardiac ultrasound images," IEEE Transactions on Ultrasonics, Ferroelectrics, and Frequency Control, vol. 56, no. 2, pp. 404-409, February 2009.

[45] T. EchoNoRMAL et al., "Ethnic-specific normative reference values for echocardiographic la and lv size, lv mass, and systolic function: The echonormal study," JACC: Cardiovascular Imaging, vol. 8, no. 6, pp. 656-665, 2015.

[46] G. D. Aquaro, G. Camastra, L. Monti, M. Lombardi, A. Pepe, S. Castelletti, V. Maestrini, G. Todiere, P. Masci, G. Giovine et al., "Reference values of cardiac volumes, dimensions, and new functional parameters by mr: A multicenter, multivendor study," Journal of Magnetic Resonance Imaging, 2016.

[47] R. M. Lang, L. P. Badano, V. Mor-Avi, J. Afilalo, A. Armstrong, L. Ernande, F. A. Flachskampf, E. Foster, S. A. Goldstein, T. Kuznetsova et al., "Recommendations for cardiac chamber quantification by echocardiography in adults: an update from the american society of echocardiography and the european association of cardiovascular imaging," Journal of the American Society of Echocardiography, vol. 28, no. 1, pp. 1-39, 2015.

[48] C. Jenkins, S. Moir, J. Chan, D. Rakhit, B. Haluska, and T. H. Marwick, "Left ventricular volume measurement with echocardiography: a comparison of left ventricular opacification, three-dimensional echocardiography, or both with magnetic resonance imaging," European heart journal, vol. 30, no. 1, pp. 98-106, 2009.

[49] U. Reiter, G. Reiter, K. Dorr, A. Greiser, R. Maderthaner, and M. Fuchsjäger, "Normal diastolic and systolic myocardial t1 values at 1.5-t mr imaging: correlations and blood normalization," Radiology, vol. 271, no. 2, pp. 365-372, 2013.

[50] S. Ryf, J. Schwitter, M. A. Spiegel, A. K. Rutz, R. Luechinger, G. R. Crelier, and P. Boesiger, "Accelerated tagging for the assessment of left ventricular myocardial contraction under physical stress," Journal of Cardiovascular Magnetic Resonance, vol. 7, no. 4, pp. 693-703, 2005. 